\title{
Electromagnetic Phenomena Reported by Near-Death Experiencers
}

\author{
Bruce Greyson, MD \\ University of Virginia \\ Mitchell B. Liester, MD \\ Monument, $\mathrm{CO}$ \\ Lee Kinsey, $\mathrm{PhD}$, LPC \\ Dallas, $T X$ \\ Steve Alsum, MDiv; and Glen Fox, PhD \\ Colorado Springs, $\mathrm{CO}$
}

\begin{abstract}
Electromagnetic (EM) aftereffects have been reported following near-death experiences (NDEs). These effects include both (a) EM actions, apparent actions by the individual on the surrounding EM environment, and (b) EM reactions, apparent reactions of the individual to the EM environment. This study investigated EM aftereffects among 216 NDErs, 54 persons who had been close to death without NDEs, and 150 persons who had never been close to death. NDErs reported both greater EM actions and greater EM reactions than did either comparison group. Among NDErs, those with higher scores on the NDE Scale reported more EM aftereffects. These findings corroborate and extend prior studies and suggest the need for controlled experiments to measure
\end{abstract}

Bruce Greyson, MD, is the Chester F. Carlson Professor Emeritus of Psychiatry \& Neurobehavioral Sciences at the University of Virginia School of Medicine, Charlottesville, VA. Mitchell B. Liester, MD, is a psychiatrist in private practice in Monument, CO. Lee Kinsey, PhD, LPC, is a counselor in private practice in Dallas, TX. At the time of this study, Dr. Kinsey was a teaching fellow in the Department of Counseling \& Higher Education, University of North Texas, Denton, TX. Steve Alsum, MDiv, is Hospice Chaplain at Pike's Peak Hospice and Palliative Care in Colorado Springs, CO. Glen Fox, PhD, is President of Fox Materials Consulting, LLC, in Colorado Springs, CO. A preliminary report of this research was presented by Dr. Liester at the 2011 annual conference of the International Association for Near-Death Studies in Durham, NC. The authors thank Kenneth Ring, PhD, and Natasha Tassell-Matamua, PhD, for their insightful comments on preliminary drafts of this manuscript. Correspondence regarding this article should be address to Dr. Greyson at the Division of Perceptual Studies, University of Virginia Health System, 210 10th Street NE, Charlottesville, VA 22902-4754; e-mail: cbg4d@virginia.edu. 
the kinds and strengths of EM fields generated or channeled by NDErs, the kinds of EM fields and devices that are affected by NDErs, and the kinds and strengths of EM fields to which NDErs react.

KEY WORDS: near-death experience, electromagnetic aftereffects, electromagnetic actions, electromagnetic reactions

Near-death experiences (NDEs) have been defined as vivid and realistic subjective experiences that occur during physiological close brushes with death, such as cardiac arrest, or in psychological situations in which death is feared imminent, such as serious accidents or illnesses. They often involve feelings of peace and joy, exceptionally lucid thought processes and perceptions, a sense of being out of the physical body, seeing an unusually brilliant light, encountering other beings and other apparent dimensions of existence, and a sense of love and wellbeing (Greyson, Kelly, \& Kelly, 2009). The incidence of NDEs in documented close brushes with death, across prospective studies in four countries, has averaged $17 \%$ when measured with a standardized instrument (Zingrone \& Alvarado, 2009).

One of the most remarkable and most thoroughly studied aspects of NDEs is their wide range of aftereffects. NDEs are typically followed by long-lasting changes in perception of self, including loss of the fear of death, strengthened belief in life after death, a new sense of purpose, and heightened self-esteem; changes in relationships with others, including increased compassion and love, greater desire to serve others, enhanced ability to express feelings, and decreased concern for material gain and social status; and changes in attitude toward life, including greater appreciation for nature, increased focus on the present, heightened spirituality, and zest for knowledge. In addition, many near-death experiencers (NDErs) report increased paranormal phenomena, such as out-of-body experiences, apparitions, extrasensory perception, precognition, healing, and mystical experiences; and changes in consciousness, such as heightened sensation, increased energy, and decreased need for sleep (Noyes, Fenwick, Holden, and Christian, 2009).

Among the physiological aftereffects that NDErs have reported are electromagnetic (EM) phenomena. These effects include malfunctions of electrical devices, both alternating current and direct current (battery-operated), including wrist watches, lights, televisions, radios, computers, appliances, vehicles, and cell phones, in close proximity to NDErs. Although EM effects were rarely reported by early research- 
ers of NDE aftereffects, they have attracted increasing attention in recent years. One reason for this increased notice may be the dramatic proliferation of electronic devices during that period. For example, quartz watches became available in the early 1970s, computers became available to the public during the mid-1980s, and cell phones gained widespread use during the mid-1990s.

Although several early researchers reported NDErs' anecdotal accounts of their EM devices malfunctioning and of their unpleasant reactions to EM fields, such reports were difficult to evaluate in the absence of comparison groups because of their frequency among nonNDErs as well. Indeed, some of these phenomena, such as malfunctioning cell phones and computers, are widely accepted by the general public as facts of life with modern technology. These early reports of EM effects following NDEs raised several questions: First, if NDErs do indeed report EM effects more frequently than do other people, is that increased incidence associated specifically with an NDE or might it be related to coming close to death, whether or not the close brush included an NDE? Second, if NDErs more frequently report EM effects, has the increase manifested only since the NDE, or might it have preceded the experience? And third, if only some NDErs report increased EM effects, what might be the relationship between nature and/or depth of NDEs and increased EM effects?

Kenneth Ring (1992) reported the first controlled study of EM effects, comparing 74 NDErs and 54 people who were interested in NDEs but had not had one themselves, with regard to electric or electronic devices (e.g., car batteries or electrical system, lights, watches, tape recorders, computers, etc.) malfunctioning in their presence. Whereas $24 \%$ of NDErs endorsed the item, only $7 \%$ of nonNDErs did, a significant difference. Ring's participants provided several narrative comments, such as the following:

I have a difficult time as many computers malfunction and lights will blow when I walk under them. This has happened for years, and I tried to ignore that this was happening. I simply cannot wear a watch for long before it breaks down. I went to ... a department store and walked in front of their brand new computer and it quit working. . . . When I [held a fluorescent light in my hands], the entire bulb lit up, like it was turned on. It seemed like there was a lot of static electricity. (p. 159)

When Ring first started looking into this phenomenon 25 years ago, he described interference primarily with wrist watches, light bulbs, and car batteries. Since that time, NDErs have reported influencing 
their cell phones, modems, fax machines, satellite radios, televisions, videocassette recorders, intercoms, vacuum cleaners, toasters, airplanes, implantable defibrillators, GPS devices, and Wii consoles. One of the most corroborated accounts came from Rebecca Stephens, Medical Director of Leesburg Hospital in Gainesville, FL, who had had a childhood NDE. She described her experience with her wrist watches:

\begin{abstract}
They either go backwards, they stop, or I have to put a new battery in every couple of months. ... I have had lots of problems with cell phones not working. I have to have them constantly changed out. . . . After I had touched the borrowed cell phone I'm using right now, because I had to send mine into the shop to get it repaired, it just beeps all day long if I touch it until I turn it off.

The IT [information technology] specialists at work would tell me I had too much static; so, I actually used static mats; I actually have something on my keyboard before I even touch my computer. Having gone through 6 hard drives in 5 years, my employers have spent some money on me to make sure I'm not full of EM energy; but in the same sense it affects everything that I do. (Bonenfant, 2005)
\end{abstract}

In a more recent and more detailed study, Farnoosh Nouri and Janice Miner Holden (2008) compared 37 NDErs, 20 nonNDErs who reported a close brush with death, and 46 people who reportedly had not been close to death, in the incidence of EM effects, narrowing the list to the four devices they seemed to report most frequently: lights, watches, computers, and cell phones. They developed 2 to 5 Likert scale items, some worded positively and some negatively, for each category. The NDErs reported significantly more EM effects than did both comparison groups. Furthermore, the higher the score on the NDE Scale, the more EM effects were reported. Some NDErs reported noticing a relationship between their EM effects and their physical and emotional states, with conditions such as fatigue and emotional arousal exacerbating the effects; and some reported having positive effects on computers and other EM devices, such as broken EM devices operating normally in their presence.

Cheryl Fracasso and Harris Friedman (2012) reported preliminary results of a study of 136 NDErs, of whom 71\% reported EM phenomena. Their report included extensive narrative accounts by NDErs of their EM aftereffects, but at the time of this preliminary report, they had not completed the third phase of their study exploring EM effects among a nonNDEr comparison group.

Sarah Blalock, Holden, and P. M. H. Atwater recently surveyed the literature on EM aftereffects of NDEs (2016). In an effort to bring 
order to the confusing array of studies, they distinguished between environmental effects that clearly involve electromagnetism and those that may or may not do so, such as biological and psychosocial effects. They further differentiated between EM actions, phenomena in which the person acts on the environment, and EM reactions, those in which the person reacts to the environment.

The data collected so far suggesting that NDErs report more EM phenomena than other people raise several questions, the primary ones being whether such reports can be corroborated by objective observations and how they can best be understood. For example, are increased EM effects present in people who have NDEs only after their NDEs or also prior to their NDEs? That is, do their NDEs somehow result in unusual EM profiles, or did their unusual EM profiles make them more likely to have NDEs-or did some other trait lead to both their EM effects and to NDEs?

In addition, there are questions about methodological artifacts in these surveys. For example, as Nouri and Holden (2008) noted, it is conceivable that NDErs may report more EM effects simply because they use less reliable electronic devices than do other people, because they use EM devices more than do other people, or because they have less expertise with EM devices than do other people. We consider these possibilities unlikely, as did Nouri and Holden (2008), and we know of no evidence supporting any of them, but they are not entirely impossible.

Finally, around $70 \%$ of the NDErs in the studies by Nouri and Holden (2008) and by Fracasso and Friedman (2012) reported EM effects, compared to only a quarter of those in Ring's study (1992). Although that increased prevalence of EM effects in recent studies may be related to the amount of EM pollution in the environment stemming from the omnipresence of smartphones and other electronic media (K. Ring, e-mail communication, February 26, 2016), the discrepancy in rates may also reflect a difference in sampling technique, a difference in how questions were asked, or an increase in NDErs' willingness to acknowledge these effects, rather than an increase in actual EM effects of the intervening years.

We attempted to address some of these questions with an extensive survey of NDErs recruited from among people who volunteered to share their NDE accounts and from those attending support groups for NDErs, comparing their responses with nonNDErs recruited through an online survey. For the sake of consistency, we will use Blalock, Holden, and Atwater's terms EM action for apparent effects 
of the individual on the electromagnetic environment and EM reaction for apparent responses of the individual to the electromagnetic environment.

\section{Methods}

\section{Participants}

Our sample of 420 participants included 216 persons (51\%) who reported having had NDEs that scored 7 or more points on the NDE Scale (see below), and 204 persons (49\%) who either denied ever having been close to death or reported an experience near death that scored fewer than 7 points on the NDE Scale (nonNDErs). Of the 204 nonNDErs, 54 (26\% of the nonNDErs; $13 \%$ of the total sample) reported having been close to death without an NDE and 150 (74\% of the nonNDErs; $36 \%$ of the total sample) denied ever having been close to death.

The participants were recruited from three different sources: 143 (34\%) came from a pool of persons who had spontaneously contacted the first author to share their accounts of their experiences when they had come close to death, 72 (17\%) were recruited through Friends of the International Association for Near-Death Studies (IANDS) local support groups, and 205 (49\%) responded to an online survey (described below). Among those 143 participants recruited from the pool of persons who had volunteered to share their accounts of a brush with death, 91\% had had NDEs and 9\% had not. Among those 72 recruited from Friends of IANDS groups, $76 \%$ had had NDEs, $4 \%$ had been close to death without NDEs, and $19 \%$ had never been close to death. Among the 205 online survey respondents, 15\% had had NDEs, 19\% had been close to death without NDEs, and $66 \%$ had never been close to death.

Looking at the participants another way, among those 216 who had had NDEs, $60 \%$ came from the pool of persons who had volunteered to share their experiences close to death, $26 \%$ were recruited from Friends of IANDS groups, and 14\% were online survey respondents. Among those 54 who had been close to death without NDEs, 24\% came from the pool of persons who had volunteered to share their experiences close to death, 6\% were recruited from Friends of IANDS groups, and $70 \%$ were online survey respondents. Among those 150 who had never been close to death, $9 \%$ were recruited from Friends of IANDS groups and $91 \%$ were online survey respondents.

As noted in Table 1, the 216 near-death experiencers and the 204 
Table 1. Demographic and Historical Variables

\begin{tabular}{|c|c|c|c|c|c|c|}
\hline Variable & $\begin{array}{c}\text { NDErs } \\
(n= \\
216)\end{array}$ & $\begin{array}{c}\text { Near } \\
\text { death, } \\
\text { without } \\
\text { NDE } \\
(n=54)\end{array}$ & $\begin{array}{c}\text { Never } \\
\text { near } \\
\text { death } \\
(n=150)\end{array}$ & Statistic & $p$ & $\begin{array}{c}\text { Effect } \\
\text { size }\end{array}$ \\
\hline Female & $70 \%$ & $56 \%$ & $73 \%$ & $\chi^{2}(\mathrm{df}=2)=5.77$ & .07 & $V=.12$ \\
\hline $\begin{array}{l}\text { College } \\
\text { education }\end{array}$ & $62 \%$ & $66 \%$ & $64 \%$ & $\chi^{2}(\mathrm{df}=2)=5.04$ & .46 & $V=.08$ \\
\hline $\begin{array}{l}\text { Age at brush } \\
\text { with death }\end{array}$ & $28.5 \mathrm{yrs}$ & $32.5 \mathrm{yrs}$ & & $t(\mathrm{df}=268)=-1.77$ & .13 & $d=.22$ \\
\hline Age now & $64.7 \mathrm{yrs}$ & $53.3 \mathrm{yrs}$ & $47.5 \mathrm{yrs}$ & $F(\mathrm{df}=2,417)=10.83$ & $<.001$ & NA \\
\hline $\begin{array}{l}\text { Time since } \\
\text { brush with } \\
\text { death }\end{array}$ & $33.2 \mathrm{yrs}$ & $20.8 \mathrm{yrs}$ & & $t(\mathrm{df}=268)=5.13$ & $<.001$ & $d=.63$ \\
\hline NDE Scale & 17.4 & 2.8 & & $t(\mathrm{df}=268)=16.48$ & $<.001$ & $d=2.01$ \\
\hline $\begin{array}{l}\text { Operated EM } \\
\text { equipment }\end{array}$ & $15 \%$ & $22 \%$ & $9 \%$ & $\chi^{2}(\mathrm{df}=2)=6.98$ & .03 & $V=.13$ \\
\hline $\begin{array}{l}\text { Cardiac } \\
\text { resuscitation }\end{array}$ & $30 \%$ & $30 \%$ & & $\chi^{2}(\mathrm{df}=2)=6.65$ & .95 & $V=.00$ \\
\hline $\begin{array}{l}\text { Electrical } \\
\text { cardioversion }\end{array}$ & $19 \%$ & $19 \%$ & & $\chi^{2}(\mathrm{df}=2)=0.00$ & .99 & $V=.00$ \\
\hline $\begin{array}{l}\text { Other electri- } \\
\text { cal shock }\end{array}$ & $15 \%$ & $11 \%$ & & $\chi^{2}(\mathrm{df}=2)=0.63$ & .43 & $V=.05$ \\
\hline Cardiac arrest & $32 \%$ & $17 \%$ & & $\chi^{2}(\mathrm{df}=2)=14.32$ & .001 & $V=.23$ \\
\hline CNS trauma & $55 \%$ & $19 \%$ & $7 \%$ & $\chi^{2}(\mathrm{df}=2)=89.00$ & $<.001$ & $V=.46$ \\
\hline $\begin{array}{l}\text { Exposure to } \\
\text { EM fields }\end{array}$ & $33 \%$ & $19 \%$ & $11 \%$ & $\chi^{2}(\mathrm{df}=2)=22.38$ & $<.001$ & $V=.24$ \\
\hline
\end{tabular}

non-experiencers were statistically comparable in terms of gender and education. Among those participants who had come close to death, those who reported NDEs were older than those who did not, and they reported a brush with death longer ago than those who did not; their ages at the times of their close brushes with death were comparable. As required by the definition of group status by NDE Scale score, the near-death experiencers had significantly higher scores $($ mean $=$ $17.4 \pm 6.4)$ than did the non-experiencers who had come close to death (mean $=2.8 \pm 2.2)$. Cohen's effect size value $(d=2.01)$ reflected an extremely large effect. 
In terms of their prior experiences that may have predisposed participants to have EM effects, the NDErs reported more exposure to EM fields, more central nervous system trauma, and more cardiac arrest than did the nonNDErs. However, despite the difference in cardiac arrest rates, the two groups were statistically comparable in terms of having been resuscitated and having experienced electrical cardioversion, as well as having been exposed to other kinds of electrical shock. NDErs reported somewhat less experience operating high power electrical or magnetic equipment or heavy machinery than did nonNDErs who had been close to death, but more than those nonNDErs who had never been near death.

\section{Procedure}

As noted above, participants in this study were recruited from three different sources. First, those participants who had volunteered to share with the first author their experiences near death were mailed or e-mailed a questionnaire that included demographic data and details of their close brush with death; the standardized, self-rated NDE Scale (Greyson, 1983); and the Electromagnetic Phenomena Questionnaire developed for this study by the authors. Second, to supplement the participant pool, the second author contacted the facilitators of Friends of IANDS support groups, and asked them to distribute this questionnaire to group members willing to complete it. Participants in those two groups completed these questionnaires at a time and place of their choosing and returned them by mail or e-mail. Both those sources yielded a large number of NDErs and a smaller number of nonNDErs as well. Third, in order to recruit a comparably sized sample of nonNDErs, the third author solicited participants from the general population through Qualtrics, a private research software company that facilitates online data collection (http://www.qualtrics.com/).

In order to get a nationally representative sample, we converted the original paper questionnaire to an electronic version via the Qualtrics software. The format of some questions was altered to adhere to electronic formats provided by the software and to eliminate redundancy. For example, respondents who indicated that they did not have difficulty wearing watches were diverted by the Qualtrics software away from subsequent questions regarding the nature of the difficulty in wearing watches. In most cases, we transcribed the exact wording from the paper copy to the electronic copy, but some extraneous instructions from the paper copy were no longer required as a result 
of the software's ability to guide the participant to the next relevant question. At the suggestion of a Qualtrics representative, we added an attention filter question to help eliminate from the sample those respondents who randomly answered questions and a question to indicate the respondent's gender.

We contracted with representatives from Qualtrics who provided a nationally representative panel of respondents. As part of their policy, they guaranteed quality responses from a national sample they recruited independently. We stipulated only that we receive at least 200 responses and that the sample coincide with the 70:30 female to male ratio from our NDEr sample. Qualtrics utilized Paradigm Precision Sample (PPS; https://paradigmsample.com/), a separate company, which recruited individuals to answer the survey Qualtrics administered. PPS offered incentives to individuals in order to recruit them to their respondent panels. They recruited respondents by placing ads on high traffic sites, through the websites of business partners, and through volunteers who visited the PPS website. Once people signed up to become potential respondents to surveys, PPS collected demographic data that enabled them to place them on specific panels. Potential respondents were invited to participate in our survey via an email from PPS that did not include any information on its topic, length, or possible incentives. Once completing the survey, respondents were given the option to be rewarded with points they could use to buy products through the PPS program or with a donation to a charitable organization on the respondent's behalf.

Qualtrics kept records of all surveys that were started, a total of 835. Qualtrics designated which surveys should not be included in our analyses because they were not complete, did not pass the attention filter, or did not meet the 70:30 female-to-male ratio; together, these categories comprised a total of 630 surveys. Thus, we included in our analysis the 205 surveys (25\% of all surveys that had been started) that met completion, attention filter, and sex ratio criteria.

The study protocol was approved by the University of Virginia's Institutional Review Board for Social and Behavioral Sciences and by the University of North Texas Institutional Review Board.

\section{Measures}

NDE Scale. The NDE Scale (Greyson, 1983), a self-rated, 16-item, multiple-choice questionnaire, was used to assess near-death experiences. It has been shown to differentiate NDEs from other close 
brushes with death (Greyson, 1990); and to have high internal consistency, split-half reliability, and test-retest reliability over a short-term period of 6 months (Greyson, 1983) and over a long-term period of 20 years (Greyson, 2007). A Rasch rating-scale analysis established that the NDE Scale yields a unidimensional measure, invariant across gender, age, intensity of experience, or time elapsed since the experience (Lange, Greyson, and Houran, 2004). Although the NDE Scale was developed as an ordinal scale without quantified anchor points, the fact that it satisfactorily fits the Rasch model suggests that, for all practical purposes, there do appear to be equal distances between the points of measurement that give the scale interval-level measurement properties (Wright \& Masters, 1982).

Electromagnetic Phenomena Questionnaire. The Electromagnetic Phenomena Questionnaire developed for this study addressed a variety of effects that may or may not be related to EM fields and that have previously been reported by NDErs. It included 38 questions (some of them multi-part) regarding malfunctioning of wrist watches, light bulbs, radios and televisions, batteries, electrical appliances, cell phones, and other electronic devices in the presence of the individual; and sensations of light or color, humming or other noises, vibration or touch, nausea or dizziness, and other sensations that the individual experienced in the presence of external EM fields. Because EM effects have been associated anecdotally with other purported NDE aftereffects such as apparent psychokinesis and healing abilities, which are not clearly related to EM forces, the questionnaire also included questions about "any other new abilities" since their close brush with death, such as positive and negative effect of the individual on other people's emotions or physical symptoms. Respondents were encouraged to supplement their short answers with narrative elaborations or explanations.

\section{Statistical Analysis}

For analyses of demographic and historical variables, we used chisquared tests to assess differences between groups in terms of gender, education, history of operating EM equipment, exposure to EM fields, cardiac arrest, cardiac resuscitation, electrical cardioversion, other kinds of electrical shock, and central nervous system trauma. We used $t$ tests to assess differences between NDErs and nonNDErs in terms of NDE Scale scores, age at the time of the brush with death, and time elapsed since the brush with death. We used an analysis of 
variance to assess differences between groups in terms of present age. For analyses of EM and other effects, we used chi-squared tests to assess differences between groups in terms of individual actions and reactions reported.

In all statistical analyses, we set the threshold for statistical significance at $p<.05$. To interpret effect sizes in $t$ tests, we used Cohen's (1988) cautious guidelines. For Cohen's $d$, a value of at least .2 indicated a small, .5 a medium, and .8 a large effect. To interpret effect sizes in chi-squared tests, we used Cohen's (1988) guidelines for Cramer's $V$. For Cramer's $V$ with 1 degree of freedom (df), a value of .1 indicated a small, .3 a medium, and .5 a large effect. For Cramer's $V$ with $2 \mathrm{df}, .07$ indicated a small, .21 a medium, and .35 a large effect.

All data analyses were performed using SPSS 22 (IBM, Armonk, NY, USA).

\section{Results}

\section{Electromagnetic Actions}

Among the entire sample, apparent EM actions by the individual on the environment were reported by 67 of the 216 NDErs (31\%), by 6 of the 54 participants who had been close to death without NDEs (11\%), and by 13 of the 150 who had never been close to death (9\%). Statistically, NDErs reported significantly more EM actions than did the two comparison groups $\left(\chi^{2}=31.06, \mathrm{df}=2 ; p<.001\right)$. Cramer's $V$ for this analysis was .25 , suggesting a medium effect.

Furthermore, among the NDErs in this study, the mean NDE Scale score among those reporting EM actions was $19.3(S D=6.3)$, and that among those who did not report EM actions was $15.8(S D=6.0)$. Statistically, NDErs who reported EM actions had significantly deeper NDEs than those who did not $(t=4.13, \mathrm{df}=214 ; p<.001)$. Cohen's effect size value $(d=.57)$ suggested a medium effect.

Table 2 presents the percentages of NDErs, participants who had been near death without an NDE, and participants who had never been near death who endorsed various apparent EM actions.

Wristwatches. As noted in Table 2, NDErs reported difficulty wearing wristwatches significantly more often than did the two groups of non-experiencers, with a medium effect. They also reported wristwatches malfunctioning significantly more often, with a small to medium effect. However, the three groups did not differ significantly in terms of specific kinds of malfunction (watches stopping, watches continuing to run but showing the wrong time, and stopped watches 
Table 2. Apparent EM Actions of Individuals on the Environment

\begin{tabular}{|l|c|c|c|c|c|c|}
\hline \multicolumn{1}{|c|}{ Effect } & NDErs & $\begin{array}{c}\text { Near death, } \\
\text { no NDE }\end{array}$ & $\begin{array}{c}\text { Never near } \\
\text { death }\end{array}$ & $\chi^{2}$ (df = 2) & $\boldsymbol{p}$ & $\begin{array}{c}\text { Effect } \\
\text { size }\end{array}$ \\
\hline $\begin{array}{l}\text { Difficulty wearing } \\
\text { wristwatch }\end{array}$ & $42 \%$ & $19 \%$ & $18 \%$ & 28.51 & $<.001$ & $V=.26$ \\
\hline $\begin{array}{l}\text { Wristwatch } \\
\text { malfunctions }\end{array}$ & $34 \%$ & $20 \%$ & $15 \%$ & 17.22 & $<.001$ & $V=.20$ \\
\hline Wristwatch stops & $86 \%$ & $73 \%$ & $70 \%$ & 3.82 & .15 & $V=.19$ \\
\hline $\begin{array}{l}\text { Wristwatch shows } \\
\text { wrong time }\end{array}$ & $60 \%$ & $73 \%$ & $70 \%$ & 1.14 & .57 & $V=.11$ \\
\hline $\begin{array}{l}\text { Wristwatch restarts } \\
\text { later }\end{array}$ & $15 \%$ & $36 \%$ & $35 \%$ & 8.76 & .07 & $V=.20$ \\
\hline $\begin{array}{l}\text { Watch worn on left } \\
\text { wrist }\end{array}$ & $71 \%$ & $79 \%$ & $65 \%$ & 4.20 & .38 & $V=.13$ \\
\hline $\begin{array}{l}\text { Watch moved to other } \\
\text { wrist }\end{array}$ & $31 \%$ & $18 \%$ & $17 \%$ & 10.24 & .037 & $V=.22$ \\
\hline Light bulbs fail & $34 \%$ & $9 \%$ & $7 \%$ & 42.40 & $<.001$ & $V=.32$ \\
\hline Radio/TV malfunctions & $25 \%$ & $6 \%$ & $10 \%$ & 19.00 & $<.001$ & $V=.21$ \\
\hline Batteries malfunction & $13 \%$ & $2 \%$ & $5 \%$ & 9.37 & .009 & $V=.15$ \\
\hline $\begin{array}{l}\text { Electrical appliances } \\
\text { malfunction }\end{array}$ & $25 \%$ & $7 \%$ & $7 \%$ & 23.80 & $<.001$ & $V=.24$ \\
\hline $\begin{array}{l}\text { Cell phone } \\
\text { malfunctions }\end{array}$ & $21 \%$ & $4 \%$ & $7 \%$ & 18.13 & $<.001$ & $V=.21$ \\
\hline $\begin{array}{l}\text { Electronic devices } \\
\text { malfunction }\end{array}$ & $19 \%$ & $7 \%$ & $6 \%$ & 14.96 & .001 & $V=.19$ \\
\hline
\end{tabular}

restarting), possibly because the sample size for each individual kind of malfunction was too small to detect the effect. For example, one NDEr wrote:

This question cracked me up, as it set me to remembering the frustration of my parents. . . . Not a single wristwatch they gave me, no matter the price, ever worked more than 10 minutes on me after my coma experience when I was 9.

Another wrote:

They stop working properly within a month. Many times the battery is dead and electrolysis has corroded the works. I've even tried kinetic watches without batteries and glass backs. Within a year I destroyed a $\$ 500$ Seiko Kinetic; the insides were corroded and the capacitor was shot. The watch repairman said he'd never seen anything like it. 
Among those reporting difficulty wearing watches, participants reported the greatest difficulty with battery-powered watches, less difficulty with wind-up and kinetic (self-winding) watches, and least difficulty with solar-powered watches. However, the numbers were too small to permit statistical comparisons with different kinds of watches.

Approximately $70 \%$ of participants in all three groups reported wearing a wristwatch on their left wrist, but significantly more NDErs, with a medium effect, reported moving their watches to another location (e.g., the opposite wrist, a pocket, or a necklace) to reduce malfunctions.

Light bulbs. As shown in Table 2, NDErs reported light bulbs malfunctioning (e.g., blinking on and off or burning out) in their presence significantly more often than did either of the two groups of nonexperiencers, with a medium effect. One NDEr wrote: "It's not uncommon for street lights to go out when I walk under them", and he attached to his e-mailed questionnaire a video clip recorded on his smartphone of a street light repeatedly going off and then on again as he walked toward and then away from it. Another wrote: "When I'm upset and I turn on a light switch, there will be a bright flash and the bulb is burned out"; and a third wrote: "I have trouble driving at night because my headlights will start flashing on and off for no reason."

Radios and televisions. As shown in Table 2, NDErs reported radios and televisions malfunctioning in their presence significantly more often than did either of the two groups of non-experiencers, with a medium effect. One NDEr wrote: "The radio will occasionally turn itself on by itself." Another wrote: "A month after my NDE, I got into a friend's car and the radio that hadn't worked for years started to play." A third wrote: "Radios and TVs often change stations or channels when I am enjoying them a lot. It never happens when I don't care what's on, but only when I am intently listening or watching."

Batteries. As shown in Table 2, NDErs reported car batteries or other batteries malfunctioning in their presence significantly more often than did either of the two groups of non-experiencers, with a small effect. One NDEr wrote: "My car went dead when I pulled up to my wife's house to discuss our divorce. The doors locked and would not open, and I had to hand crank my moon roof open and climb out of the top." Participants reported batteries draining prematurely in cars, flashlights, cameras, insulin pumps, TV remote controls, clocks, cell phones, and electric toothbrushes; and many reported batteries malfunction, sometimes reversibly, only when they are emotionally stressed. 
Computers, tape recorders, and other electrical appliances. As shown in Table 2, NDErs reported computers, tape recorders, and other electrical appliances malfunctioning in their presence significantly more often than did either of the two groups of non-experiencers, with a medium effect. One NDEr wrote:

The morning of my son's funeral, a month after my NDE, I was trying to record three songs for his service on a cassette player. It seemed to record properly, but when I tested it, there was nothing on the tape. I changed the tape, with the same results. I changed cassette players and used new tapes, but still had the same outcome. I went through four cassettes players with the same result. I managed to record other things, including other songs, but not the three I wanted. I called a friend and asked her to bring her cassette recorder, but I still couldn't get it to record those three songs. My friend also tried while I watched, but it still wouldn't record those particular songs. In desperation, because I was going to be late for the funeral, my friend said she would keep trying while I took a shower. It worked perfectly the first time for her as soon as I left the room.

Another NDEr wrote: "I've had carpet shampooers catch on fire, hair dryers spark and burn out, ceiling fans turn on by themselves, and microwave ovens short out." A third wrote: "The instant I sat down on a stool at the diner, just feet from the main cooking grills, there was a loud click and both grills' circuit breakers flipped off. The manager looked at me and asked how I did that." Another wrote: "My presence never opens automatic electric-eye doors like at warehouse stores. I can walk back and forth in front of them, and the door won't open. I usually wait for someone else to walk up and then the door will open." Others reported malfunctions of computers, refrigerators, vending machines, elevators, electric can openers, coffee makers, tape recorders, fax machines, printers, vacuum cleaners, cash registers, automated tellers, security alarms, x-ray diffraction machines in a geological survey laboratory, and grocery store bar code scanners.

Cell phones. As shown in Table 2, NDErs reported cell phones malfunctioning in their presence significantly more often than did either of the two groups of non-experiencers, with a medium effect. One NDEr wrote: "I can't use them because of countless dropped calls, but friends of mine often have unexplained problems with reception only when I'm around." Another wrote:

While standing next to me, my friend got a phone call from my phone, which was turned off. He showed me his phone as it was ringing, and the call indeed came from my phone number. I asked him to answer 
the phone to see what I had to say. When he did, all he heard was clicking and funny hissing noises.

A third wrote: "I get mysterious text messages that are gibberish." Another wrote: "I can't type characters in a search window without it filling in the rest of the window with random letters."

Other electronic devices. As shown in Table 2, NDErs reported other electronic devices malfunctioning in their presence significantly more often than did either of the two groups of non-experiencers, with a small effect. They reported malfunctions with illuminated make-up mirrors, microphones, power screwdrivers, electric shavers, fire alarms, electronic keyboards, CPAP machines for sleep apnea, doorbells, smoke detectors, thermostat liquid crystal display (LCD) screens, mustache trimmers, and electric typewriters.

\section{Electromagnetic Reactions}

Among the entire sample, reactions of individuals to the EM environment were reported by 125 NDErs (58\%), by 11 participants who had been close to death without NDEs (20\%), and by 19 who had never been close to death (13\%). Statistically, NDErs reported significantly more EM reactions than did the two comparison groups $\left(\chi^{2}=85.71\right.$, $\mathrm{df}=2 ; p<.001)$. Cramer's $V$ for this analysis was .46 , suggesting a large effect.

Furthermore, among the NDErs in this study, the mean NDE Scale score among those who reported EM reactions was $19.2(S D=6.2)$, and that among those who did not report EM reactions was 14.8 $(S D=5.8)$. Statistically, NDErs who reported EM reactions had significantly deeper NDEs than those who did not $(t=5.28$, df $=214$; $p<.001)$. Cohen's effect size value $(d=.73)$ suggested a medium effect.

When those participants who reported sensing the presence of strong EM fields when others around them did not were asked about how they sensed the EM fields - that is, which sensory modality was involved-they reported several modalities. Visually, participants mentioned seeing white light, "a weird light," halos, waves and undulating forms, and colors becoming blurry. Auditorily, participants mentioned ringing in the ears, humming, and crackling and crunching sounds. Kinesthetically, participants mentioned heart poundings, body vibrating, goose bumps, tingling, physical cringes, "little shocks across my body, with sensations of skin crawling," "feeling like I could tip over," "sensations around my pacemaker," "feeling of a wave going 
through me," pressure in the forehead, headaches, hairs raising on the neck or arms, cold sensations, and floating sensations. Interoceptively, participants mentioned nausea, dizziness, lightheadedness, and queasiness. Participants mentioned other sensations including pain, shaking hands, dropping things, aromas, a burnt smell, a sweet smell, tastes, "a menthol breath, like peppermint breath," sleeplessness, "a need to eat crunchy things like celery or carrots," anxiety, and agitation. The numbers reporting individual modalities were too small to permit statistical comparisons.

\section{Other Electromagnetic Phenomena}

Other EM phenomena not specifically mentioned above were reported by 69 NDErs (32\%), by 6 participants who had been close to death without NDEs (11\%), and by 13 who had never been close to death (9\%). Statistically, NDErs reported significantly more "other EM phenomena" than did the two comparison groups $\left(\chi^{2}=31.82\right.$, df $=2$; $p<.001)$. Cramer's $V$ for this analysis was .28 , suggesting a medium effect.

Furthermore, among the NDErs in this study, the mean NDE Scale score among those who reported other EM phenomena was 19.3 $(S D=6.3)$, and that among those who did not report other EM phenomena was $15.8(S D=6.0)$. Statistically, NDErs who reported other EM phenomena had significantly deeper NDEs than those who did not $(t=4.13, \mathrm{df}=214 ; p<.001)$. Cohen's effect size value $(d=.57)$ suggested a medium effect.

In response to the question about other EM phenomena, participants reported a wide variety of phenomena that, in fact, may or may not involve electromagnetism. Although this question did not differentiate between EM or non-EM actions and reactions, we organized responses into these two categories. Actions that participants described included moving Styrofoam cups they sat near, erasing information encoded on magnetic stripe cards ("swipe cards"), overhead telephone wires humming when they walk by, stopping and restarting the flow of sand grains in an hourglass by concentrating on it, spinning paper pinwheels by walking nearby, and reversing the polarity of compasses. Reactions that participants described included perceiving an imminent power outage minutes before it happens, getting a headache seconds before someone nearby has a seizure, tasting metal in the mouth when holding coins, feeling agitated just before an earthquake, hearing telephones ring a few seconds before anyone else hears them, perceiving police radar traps miles away, and locating water by dowsing. 


\section{Onset of EM Phenomena}

Among the NDErs who reported EM phenomena in this study, $72 \%$ reported that these phenomena began after their NDEs, 15\% reported that they began prior to their NDEs, and 13\% could not say with any certainty when they began.

\section{Explanation for Electromagnetic Effects}

In response to a question as to why they experienced these EM phenomena, 76 of the 216 NDErs (35\%) reported having some explanation or idea, compared to 4 of the 54 participants who had been close to death without NDEs (7\%) and 11 of the 150 participants who had never been near death (7\%). Statistically, NDErs offered explanations for their purported EM effects significantly more often than did either of the two groups of non-experiencers $\left(\chi^{2}=47.78, \mathrm{df}=2 ; p<.001\right)$. Cramer's $V$ for this analysis was .34, suggesting a medium to large effect.

Several NDErs offered as an explanation simply that their own EM field had changed as a result of their NDEs, without stating any mechanism (e.g., "I was rewired"; "My energy level was increased and sometimes leaks out"; "I now vibrate to a higher frequency, interfering with electric vibrations around me"). Some mentioned their energy fields becoming "aligned" with external fields (e.g., "I have some feeling of connecting with electrical or mechanical objects that are malfunctioning"). Others stated that their NDEs increased their sensitivity to many external influences, including but not limited to EM fields (e.g., "I awakened"; "I now see all things as energy in motion and all connected"; "After dying, my mind began to understand the energetic route in and out of the body, and I'm now able to open and close those energetic doorways"). Still others offered by way of explanation not the mechanism that brought it about, but, rather, an end result for which it was brought about, such as "to give comfort to others" or "to maintain contact with the realm of spirit entities."

\section{Role of Encountering Light in the NDE}

Several NDErs associated their EM aftereffects with having encountered a brilliant light in their NDEs, the unstated assumption being that, because physical light is EM radiation within a certain portion of the spectrum, perhaps the light encountered in the NDE is also an EM phenomenon that somehow altered the experiencer's EM field. To explore this hypothesis, we examined the rate of EM effects in partici- 
pants who reported encountering light in their NDEs and those who did not.

The data were somewhat ambiguous, but did not generally support an association between encounters with light in the NDE and EM effects. Some effects, such as problems with electrical appliances and other EM devices, and sensitivity to EM fields, were reported more often by those who had encountered light in the NDE than by those who did not; but other effects, such as problems with wristwatches, light bulbs, radios or televisions, batteries, or cell phones showed no such association. Moreover, it may be that these apparent associations with an encounter of light in the NDE are spurious effects of the high correlation between encounters with light and overall depth of NDE as measured by the NDE Scale $(r=.702, n=270 ; p<.001)$. Indeed, when NDE Scale score was entered into the chi-squared analysis as a control variable, none of the EM effects remained significantly associated with encountering light in the NDE. If so, then encounters with light may have no more influence over subsequent EM effects than do other features of the NDE.

\section{Actions Not Clearly Electromagnetic}

Our questionnaire did not ask specifically about actions by the individual on the environment that are not clearly electromagnetic. However, as noted above, because EM effects have been associated anecdotally with other purported NDE aftereffects such as apparent psychokinesis and healing abilities, the questionnaire also included questions about "any other new abilities" since their close brush with death.

Positive actions. As shown in Table 3, NDErs reported changes since their close brushes with death that positively affected others' emotions significantly more often than did participants who had come close to death without NDEs, with a medium effect. The most frequent description offered was an ability to calm other people, or even animals. For example, one NDEr wrote: "People say that they feel peaceful and calm in my presence. Many people cry and feel as if a burden has been lifted. They feel lighter." Other common responses were an ability to make people feel happy, or to make them feel safe and at peace. One NDEr wrote: "People say I light up a room."

NDErs also reported a new ability to heal other people since their close brushes with death significantly more often than did participants who had come close to death without NDEs, with a medium ef- 
Table 3. Apparent Actions of Individuals on the Environment That Are Not Clearly EM

\begin{tabular}{|l|c|c|c|c|c|}
\hline \multicolumn{1}{|c|}{ Effect } & NDErs & $\begin{array}{c}\text { Near death, } \\
\text { no NDE }\end{array}$ & $\boldsymbol{\chi}^{\mathbf{2}} \mathbf{( d \mathbf { f } = 1 )}$ & $\boldsymbol{p}$ & $\begin{array}{c}\text { Effect } \\
\text { size }\end{array}$ \\
\hline $\begin{array}{l}\text { Positive effect on others' } \\
\text { emotions }\end{array}$ & $59 \%$ & $19 \%$ & 28.23 & $<.001$ & $V=.32$ \\
\hline Heal others & $41 \%$ & $6 \%$ & 25.26 & $<.001$ & $V=.31$ \\
\hline Other positive effects & $52 \%$ & $9 \%$ & 31.84 & $<.001$ & $V=.35$ \\
\hline $\begin{array}{l}\text { Negative effect on others' } \\
\text { emotions }\end{array}$ & $15 \%$ & $2 \%$ & 7.04 & .008 & $V=.16$ \\
\hline Cause illness, pain & $9 \%$ & $0 \%$ & 5.27 & .022 & $V=.14$ \\
\hline Other negative effects & $16 \%$ & $2 \%$ & 8.01 & .018 & $V=.18$ \\
\hline
\end{tabular}

fect. Some NDErs reported becoming professional healers after their NDEs, e.g., "I am a Reiki master and do energy healing." More commonly, NDErs reported using their healing abilities informally; e.g., "I can relieve pain by touching"; "I have the gift of healing others using energy work"; and "I can make medical diagnoses and have the ability to activate the body's inner healer." Some gave specific examples, such as: "Of three terminal cancer clients I used my healing energy on, all are in remission. One man's cancer disappeared from his lungs. I spotted the beginnings of a tumor in another's kidney before symptoms were manifest." Another NDEr wrote:

I could hold my hands above my daughter's shins (she was born with club foot muscles) and she would get relief from pains and aches of her shin muscles. Also, if I would hold my hands above her skin in one spot for a minute, there would by a sunburned handprint on her skin that would be there for about 10 hours.

One NDEr gave an example of healing animals:

A cat with feline leukemia adopted me. When I took him to the vet, she said he had only a few months to live; but instead he lived more than 13 years. Neighbors have asked me to help them with their pets, and they have told me they were certain I increased the pet's wellness and life span.

NDErs also reported changes since their close brushes with death that affected others positively in some other way significantly more often than did participants who had come close to death without NDEs, with a medium effect. One NDEr wrote: "People feel the energy from 
me when I am near them." Another wrote: "People tell me they have more energy when they are with me, and they feel more like getting things done. I fire them up!" A third wrote simply: "People feel good around me." Others wrote that people in distress seem to trust them.

Negative actions. Again, as shown in Table 3, NDErs reported changes since their close brushes with death that negatively affected others' emotions significantly more often than did participants who had come close to death without NDEs, with a small effect. The most common response that NDErs reported was that others felt uncomfortable with the NDErs' perceived insight into them. For example, one NDEr wrote: "I make some people nervous and uncomfortable. They can't stand to be around me because they think I am reading their minds. They think I am evil." Another wrote: "Often people 'feel' my 'reading' of their essences and avoid contact." A third wrote: "Other people become uncomfortable when they think I see or know what they don't want others to know." Some NDErs reported that other people are influenced by the NDErs' unpleasant emotions (e.g., "I can feel scared or nervous and, without saying anything, both other people and animals react with the same emotions I am having").

NDErs also reported a new ability to cause illness or pain in other people since their close brushes with death significantly more often than did participants who had come close to death without NDEs, with a small effect. Most commonly, they reported that they seem to drain other people of energy. For example, one NDEr wrote: "Two men, both of whom do not know me well and do not know each other, have experienced unpleasant effects after briefly meeting me, and complained that I was draining their energy away from them, like a vampire!" Another wrote: "People fall asleep when they visit my home and when they talk on the phone with me." Another NDEr wrote that she was able to control other people's bodies, which sometimes caused them pain.

NDErs also reported changes since their close brushes with death that affected others negatively in some other way significantly more often than did participants who had come close to death without NDEs, with a small effect. The effect mentioned most often was other people being negatively affected by the NDEr's insights. For example, one NDEr wrote: "Some people who are totally unaware of their own inner life have reacted negatively to me." Another wrote:

I had to learn when to share the presence of others. I had thought everyone would be comforted and happy to know their loved ones who 
have crossed over were still around them, sometimes offering their love and support. But I soon learned that not everyone is comforted knowing this.

Another frequent response was deteriorating family relationships because of the NDEr's closeness to others (e.g., "Closeness to soulmates not in my family has made communication with loved ones very difficult and at times impossible, resulting in very strained relationships with my husband"). NDErs also wrote about overwhelming other people energetically (e.g. "I am a 'powerhouse,' so I've had to learn to downscale that power because it is overwhelming to them"; "They don't like me because I can keep going and going and going, making them look bad"); and about unusual attractions and repulsions (e.g., "Some people are unreasonably attracted to me and some out of the blue are repelled"; "Some people tell me I have bad vibes"). One NDEr wrote about a negative effect of apparent psychokinesis: "Once, I focused very strongly while playing golf, causing the other party to knock three balls in the water in a row. I believe it was my concentration that caused this").

\section{Reactions Not Clearly Electromagnetic}

Again, our questionnaire did not ask specifically about reactions by the individual to aspects of the environment that are not clearly electromagnetic. However, in response to the questions about "any other new abilities" since their close brushes with death, some participants offered narrative responses involving their sensitivity to other people's emotions or physical symptoms.

The most common response was an increase in empathy and ability to feel other people's emotions, e.g., "I am much more empathetic, at times paralyzingly so." Other common responses included sensing energy (e.g., "ability to feel, sense, or read human energy"); seeing auras (e.g., "I see light and colors around each person and it tells me about who they are inside of themselves"); increased intuition and insight (e.g., "I have the gift of intuition, and seem to have a deeper insight into people's problems and helping them see the situation more clearly"). Others mentioned seeing the future (e.g., "I have many precognitive dreams about events that I eventually walk into") and seeing the deceased (e.g., "I sense and sometimes physically see people who have crossed over"). A few reported unpleasant reactions, for example, "I can feel negative presences around me and I take that always as a 
warning sign to get away"; "I am overly sensitive to people, and their vibrations often overwhelming me and causing nausea for me."

\section{Possible Confounding Factors in This Survey}

Age and elapsed time. Although NDErs and nonNDErs in this study did not differ in age at the time of their brushes with death, the NDErs were significantly older at the study participation time than were the nonNDErs, and accordingly, significantly more time had elapsed since the close brush with death for the NDErs than for the nonNDErs. Those differences raised the possibility that the reason NDErs reported more EM phenomena was simply that they had lived longer than the nonNDErs and, particularly, had lived longer since the brush with death. To test these hypotheses, we examined the effects of age and elapsed time on EM phenomena without regard to NDE status.

Those participants who reported EM effects had a mean age of 64.3 years $(S D=6.0)$, and those who did not report EM effects had a mean age of 61.2 years $(S D=1.2)$. That difference was not significant, with no effect $(t=0.58, \mathrm{df}=418 ; p=.56 ; d=.01)$. Likewise, those participants who reported EM effects had a mean elapsed time since the brush with death of 31.1 years $(S D=1.4)$, and those who did not report EM effects had a mean elapsed time of 30.3 years $(S D=1.4)$. That difference was also not significant, with no effect $(t=0.39$, $\mathrm{df}=268$; $p=.70 ; d=.00)$.

Participant recruitment source. The majority of NDErs in this study were either persons who had spontaneously contacted the first author to share their accounts of their experiences near death or recruits from Friends of IANDS local support groups, whereas the majority of nonNDErs were online survey respondents. Could the differences found between NDErs and nonNDErs be related to the source of the participants rather than to their status as NDErs on nonNDErs per se? This hypothesis seemed rather unlikely to us, because one might expect online survey respondents, who were anonymous, to report more anomalous phenomena than people identified by personal contact, even if by mail or e-mail. However, contrary to that expectation, the online survey respondents reported fewer EM phenomena than did those who volunteered to share their experiences or those in the Friends of IANDS groups. Nevertheless, to test for this possibility, we compared the questionnaire responses of the NDErs from the three different recruitment sources. 
As expected, there were no significant differences, with a small effect, in responses of NDErs based on their source. EM effects were reported by $45 \%$ of the NDErs who had volunteered to share their experiences, by $58 \%$ of the NDErs recruited from Friends of IANDS groups, and from $48 \%$ of the NDErs who responded to the online survey $\left(\chi^{2}=2.70, \mathrm{df}=2 ; p=.26 ; V=.11\right)$.

\section{Discussion}

Both EM actions and EM reactions were reported by NDErs in this study three times as often as by nonNDErs, whether or not those nonNDErs had ever been close to death. Furthermore, the deeper the NDE, the more likely one was to report EM actions and reactions. This pattern held true for every kind of EM action or EM reaction probed, as well as for "other EM phenomena" that participants felt did not fit into either category.

These findings corroborate and extend prior controlled studies of EM phenomena in NDErs and nonNDErs by Ring (1992), Nouri and Holden (2008), and Fracasso and Friedman (2012), as well as other researchers' uncontrolled studies and anecdotal reports. Indeed, no researcher who has investigated the question has failed to find more reported EM phenomena from NDErs than from nonNDErs and to find EM effects that increase with NDE depth. The proportion of NDErs reporting EM effects in our study was close to that of Ring's original study (1992), and substantially smaller than the more recent studies of Nouri and Holden (2008) and Fracasso and Friedman (2012). This result leads us to suspect that the different frequencies reported by various researchers may have been due more to differences in how questions were asked, rather than to an increase over the years either in actual EM effects or in NDErs' willingness to acknowledge these effects. Most investigators up to this point have developed their own questionnaires to explore EM phenomena, making it difficult to compare prevalence across studies. For this reason, it would be preferable for future researchers to agree upon one standardized questionnaire.

As in prior research, EM phenomena in this study were reported overwhelmingly to be aftereffects of NDEs: three-fourths of NDErs noted these phenomena starting only after the NDE, and only $15 \%$ noted them prior to the NDE.

Some NDErs reported constant EM actions, such as the participant who reported that her presence never opens automatic electriceye doors like at warehouse stores. On the other hand, other NDErs 
reported EM effects that appeared to be activated only under stress, such as the participant who reported that she was unable to record songs for her son's funeral. This result raises the question of whether steady and episodic EM effects are in fact different phenomena, or are variants of the same process. Future researchers might investigate this distinction.

\section{Possible Explanations}

Biomedical researchers have been reporting EM reactions for decades, although these reports have received little notice and have been regarded as controversial in the medical literature (Choy, Monro, \& Smith, 1986). Patrick Levallois (2002) described a syndrome of hypersensitivity to EM fields that manifested primarily in skin disorders (redness, dryness, itching, and burning). He and his colleagues found EM hypersensitivity among $3 \%$ of the general population in California (Levallois, Neutra, Lee, \& Hristova, 2002), and a survey of the Swedish population found a comparable incidence of $1.5 \%$ (Hillert, Berglind, Arnetz, \& Bellander, 2002).

Laboratory testing of normal people who did not claim EM sensitivity found that weak EM fields evoked electrical responses from the occipital lobe of the brain, suggesting that even humans who do not report symptoms of EM sensitivity have a biological magnetic sense (Carrubba, Frilot, Chesson, \& Marino, 2007). However, the degree of EM sensitivity among people who report being sensitive does not appear to be different from that of people who report no sensitivity (Schröttner, Leitgeb, \& Hillert, 2007); and there are ambiguous data regarding whether people who complain of EM hypersensitivity can reliably differentiate active from sham EM fields in blind experiments, with some studies yielding positive results (e.g., McCarty, Carrubba, Chesson, Frilot, Gonzalez-Toledo, \& Marino, 2011; Rea, Pan, Yenyves, Sujisawa, Suyama, Samadi, \& Ross, 1991) and others negative (e.g., Rubin, Nieto-Hernandez, \& Wessely, 2010). However, people who report EM hypersensitivity, even when they are not consciously aware of the presence of an EM field, show an altered cortical excitability not seen in the brains of people without EM hypersensitivity (Landgrebe, Hauser, Langguth, Frick, Hajak, \& Eichhammer, 2007).

There have been fewer studies of EM actions, and we know of none that have focused on people who report unintended EM effects. In research with unselected participants, EM radiation emitted from the human body has been detected in the extremely low frequency (ELF) 
range (Lipkova \& Cechak, 2005), and weak emission of photons, particles of EM radiation, from the skin has been detected in darkrooms, not associated with changes in skin temperature (Kobayashi, Kikuchi, \& Okamura, 2009). People who claimed healing abilities, but not comparison groups who did not, were able reliably to imprint some form of radiation into distilled water that modified the physical characteristics of the water as measured by electrophotography (Berden, Jerman, \& Skarja, 1997). So there is considerable evidence that at least some people's bodies respond, with or without conscious awareness, to external EM fields, and some data suggesting that at least some human bodies emit EM radiation. What remains unexplored is the relationship of these phenomena to NDEs.

Some explanation for these EM aftereffects was offered by NDErs in this study five times as often as by nonNDErs. That difference may reflect the fact that EM phenomena were sufficiently frequent and/or sufficiently disruptive in the lives of NDErs to motivate them to give these effects serious thought and to try to understand them, whereas EM phenomena may have been so unusual or mild in the lives of nonNDErs that they were able to dismiss them as meaningless events not worth further thought.

In the most commonly offered explanations, NDErs regarded EM phenomena not as unique effects but rather as one aspect of a global change in energy or in sensitivity that was manifested both in EM phenomena and in other energetic changes. Ring (1992) had reached a similar conclusion as a result of his research - that EM sensitivity was part of a larger complex of sensitivities to which NDErs are proneand Fracasso and Friedman (2012) found that those NDErs who reported EM effects also reported higher rates of allergies, chemical sensitivities, geomagnetic sensitivities, and paranormal experiences.

Actions and reactions that were not clearly EM were also reported by NDErs in this study three times as often as by nonNDErs, whether or not those nonNDErs had ever been close to death. These were most commonly described as calming or healing effects on other people and as becoming more sensitive to others' emotions and unconscious issues. Because the mechanism(s) of these effects are not known, it is conceivable that some of these effects that are not clearly electromagnetic may in fact turn out to be so, such as the effects on and sensitivity to other people's conditions. In support of that hypothesis, experienced non-contact therapeutic touch therapists, isolated from ground and surrounded on all sides by copper walls, have been shown to produce measurable electrical surges a thousand times greater than 
the skin potential changes seen in emotional arousal (Green, Parks, Guyer, Fahrion, \& Coyne, 1991).

Alternatively, it is conceivable that, although these other aftereffects are not in themselves electromagnetic, both they and the EM effects are caused indirectly by some other change in the individual that alters both their EM interactions and these other, non-EM interactions. For example, it is possible that increased sensitivity to and engagement with the external world could lead both to EM effects and to interpersonal effects, or that a loosening of the physiological filter that regulates the brain's access to consciousness (Kelly \& Presti, 2015) would magnify both EM and interpersonal effects, or that awareness of EM and interpersonal effects only appear to be increased among NDErs as a result of increased mindfulness following NDEs, making experiencers more attentive toward themselves and their environment and more attuned to their impact on, and responses to, the world around them (N. Tassell-Matamua, e-mail communication, February $28,2016)$.

\section{Possible Confounding Factors}

The role of light encountered in the NDE remains ambiguous, as data from this study supported an association with some EM effects but not others, and even those associations that appeared meaningful at first lost significance when statistical analyses were corrected for total NDE Scale (to which an experience of light contributed). This was a retrospective analysis based on suggestions from participants about the role of light in the NDE, rather than a planned analysis. Future researchers may wish to pursue this question, but at this point, it does not appear promising, as encounters with light in the NDE did not appear to be singularly important in leading to subsequent EM phenomena, any more than did other NDE features.

Although NDErs in this study were older than the nonNDErs, and particularly had lived longer after their brush with death, neither age nor time elapsed since the brush with death accounted for the increased reports of EM phenomena among NDErs. Likewise, although most of the NDErs in this study were identified through personal contacts, whereas most of the nonNDErs were recruited through an anonymous online survey, that difference did not account for the increased reports of EM phenomena among NDErs.

NDErs in this study did report more exposure to external EM 
fields, more central nervous system trauma, and more cardiac arrests (but not, interestingly, more electrical cardioversions) than did nonNDErs. It is plausible that these historical factors may play a role in the manifestation of EM phenomena, and that possibility should be explored in future research. On the other hand, it is also plausible that frequent and disruptive EM effects may prompt people to search their memories for, and recall, prior exposure to external EM fields and traumatic experiences.

\section{Other Limitations of This Study}

Our confidence in and interpretation of the findings of this study must be tempered by certain weaknesses inherent in any retrospective, cross-sectional study. The self-selection of participants and the reliance on retrospective self-reports of experiences and aftereffects may have limited the generalizability of these findings.

Because our sample of participants who had come close to death was self-selected, we do not know whether (or how) they may differ from other survivors of close brushes with death who chose not to participate in the study. It is conceivable that survivors of near-death events who have more EM phenomena may be more willing to participate in research than those who do not.

We relied on retrospective reports of experiences and aftereffects, which may theoretically be vulnerable to memory decay, distortion, or fabrication. A prior study of the consistency of NDE Scale scores over a period of decades suggested that passage of time did not materially influence memories of the experience itself (Greyson, 2007). However, we have no estimate of the reliability over time of descriptions of EM phenomena, and, particularly, of changes in EM effects. There is little evidence that people can recall change accurately, particularly over long periods of time (Tennen \& Affleck, 2009). There is some evidence that people retrospectively overestimate causal relationships between memorable events like NDEs and subsequent change, discounting the influence of prior or subsequent events that may be less dramatic but still potentially transformative (Tennen \& Affleck, 2009); although there is also contradictory evidence of reluctance to attribute change to a specific traumatic event (Smith \& Cook, 2004).

We can therefore conclude that having an NDE during a close brush with death is associated with the subsequent perception of more EM phenomena, but we do not have evidence bearing on differences 
in actual EM actions and reactions as measured by objective assessments. It would be helpful in future research to incorporate objective measures of EM phenomena using scientifically calibrated devices in a controlled setting.

We undertook this study with the assumption that NDEs are unitary phenomena, and indeed a Rasch rating-scale analysis of the NDE Scale lends statistical support to that unidimensional approach (Lange, Greyson, \& Houran, 2004). However, it is conceivable that different types of NDE might be differentially associated with subsequent EM phenomena. The possibility of differential effects of different types of NDE might further limit generalizability of these findings, and suggest future research aimed at elucidating the particular NDE features that may be associated with subsequent posttraumatic growth. In addition, further research is needed to ascertain whether (and how) EM effects may vary longitudinally and what individual and situational variables in the near-death event may predict EM aftereffects.

\section{Possible Future Research Directions}

As noted above, despite the prevalence of EM phenomena reported by NDErs, there has not yet been a substantial controlled experiment investigating this effect. In 1997, Jim Knittweis conducted an informal study to see whether he could detect a difference between electrical fields of NDErs and nonNDErs. He employed two devices for his tests: a thermistor for testing heat from the participants' fingers and an electronic electroscope to measure the electron flow. Knittweis tentatively concluded that most NDErs did not show differences in heat or electron flow from their hands compared to nonNDErs; however, NDErs who reported having gained healing abilities from their NDEs seemed to have more overall heat and electron flow from both hands compared to nonNDErs. Because of the absence of a blind evaluation procedure, and the failure to control other factors, such as time of day, weather conditions, and whether participants' clothes were electrostatic, these results can be considered only suggestive. Knittweis suggested, however, that it provided sufficient evidence to justify a larger scale study using scientifically calibrated devices under controlled conditions.

We believe that sufficient subjective evidence of the link between NDEs and EM effects has been collected to justify objective exploration of this association, as Knittweis proposed two decades ago. In addition to controlling for factors that are known to influence electro- 
static phenomena, it would be desirable to conduct such experiments within a Faraday cage to eliminate the influence of fluctuating environmental EM fields. Participants could be tested for their influence on different kinds of watches, such as battery-powered, solar-powered, wind-up, and automatic (self-winding); or for their influence on different kinds of light bulbs, such as incandescent, light-emitting-diode (LED), compact fluorescent (CFL), or halogen.

It is generally preferable for data to be collected automatically because that process eliminates the possible errors of perception and transcription inherent in manually collected data. However, for a study of EM effects, manual data collection and recording might be advisable in order to eliminate the prospect of the participant influencing the automated data collection mechanism rather than the target. As noted above, other aspects that may prove fruitful in future research include the association between EM effects and various NDE features such as encounters with light, the distinction between constant and stress-related EM effects, and the association between EM effects and other energetic phenomena following NDEs.

\section{Conclusion}

The findings from this study confirm and extend those from previous research and anecdotal accounts: NDErs report more EM actions on the environment and more reactions to the EM environment than do nonNDErs, both those people who had been close to death without NDEs and those who had never been close to death. Furthermore, among NDErs, the deeper the experience (as reflected in the NDE Scale), the more likely the experiencer is to report EM actions and reactions. NDErs who report EM effects also tend to report environmental effects that are not clearly electromagnetic. The mechanism of this association between EM and other effects remains ambiguous.

We echo Knittweis's belief that sufficient subjective evidence of the link between NDEs and EM effects has been collected to justify objective exploration of this association using scientifically calibrated devices in a controlled setting.

\section{References}

Berden, M., Jerman, I., \& Skarja, M. (1997). A possible physical basis for the healing touch (biotherapy) evaluated by high voltage electrophotography. Acupuncture and Electro-Therapeutics Research, 22, 127-146. 
Blalock, S., Holden, J. M., \& Atwater, P. M. H. (2016). Electromagnetic and other environmental effects following near-death experiences: A primer. Journal of Near-Death Studies, 33(4), 181-211.

Bonenfant, R. (2005, September). Electromagnetic sensitivity: A physician's experience following a childhood near-death experience. Paper presented at the International Association for Near-Death Studies Annual Conference, Virginia Beach, VA, 2005.

Carrubba, S., Frilot, C., Chesson, A. L., \& Marino, A. A. (2007). Evidence of a nonlinear human magnetic sense. Neuroscience, 144, 356-367.

Choy, R. V. S., Monro, J. A., \& Smith, C. W. (1986). Electrical sensitivities in allergy patients. Clinical Ecology, 4, 93-102.

Cohen, J. (1988). Statistical power analysis for the behavioral sciences (2nd ed.). Mahwah, NJ: Lawrence Erlbaum Associates.

Fracasso, C., \& Friedman, H. (2012). Electromagnetic aftereffects of near-death experiences: A preliminary report on a series of studies currently under way. Journal of Transpersonal Research, 4(2), 34-55.

Green, E. E., Parks, P. A., Guyer, P. M., Fahrion, S. L., \& Coyne, L. (1991). Anomalous electrostatic phenomena in exceptional subjects. Subtle Energies, 2, 69-94.

Greyson, B. (1983). The Near Death Experience Scale: Construction, reliability, and validity. Journal of Nervous and Mental Disease, 171, 369-375.

Greyson, B. (1990). Near-death encounters with and without near-death experiences: Comparative NDE Scale profiles. Journal of NearDeath Studies, 8, $151-161$.

Greyson, B. (2007). Consistency of near-death experience accounts over two decades: Are reports embellished over time? Resuscitation, 73, 407-411.

Greyson, B., Kelly, E. W., \& Kelly, E. F. (2009). Explanatory models for neardeath experiences. In J. M. Holden, B. Greyson, \& D. James (Eds.), The handbook of near-death experiences: Thirty years of investigation (pp. 213-234). Santa Barbara, CA: Praeger/ABC-CLIO.

Hillert, L., Berglind, N., Arnetz, B. B., \& Bellander, T. (2002). Prevalence of self-reported hypersensitivity to electric or magnetic fields in a populationbased questionnaire survey. Scandinavian Journal of Work, Environment and Health, 28, 33-41.

Kelly, E. F., \& Presti, D. E. (2015). A psychobiological perspective on "transmission" models. In E. F. Kelly, A. Crabtree, \& P. Marshall (Eds.), Beyond physicalism: Toward reconciliation of science and spirituality (pp. 115-155). Lanham, MD: Rowman and Littlefield.

Knittweis, J. (1997). Electrical sensitivity of near-death experiencers [Letter]. Journal of Near-Death Studies, 15, 223-225.

Kobayashi, M., Kikuchi, D., \& Okamura, H. (2009). Imaging of ultraweak spontaneous photon emission from human body displaying diurnal rhythm. PLoS One, 4(7), e6525.

Landgrebe, M., Hauser, S., Langguth, B., Frick, U., Hajak, G., \& Eichhammer, P. (2007). Altered cortical excitability in subjectively electrosensitive patients: Results of a pilot study. Journal of Psychosomatic Research, 62, 283-288.

Lange, R., Greyson, B., \& Houran, J. (2004). A Rasch scaling validation of a 'core' near-death experience. British Journal of Psychology, 95, 161-177.

Levallois, P. (2002). Hypersensitivity of human subjects to environmental elec- 
tric and magnetic field exposure: A review of the literature. Environmental Health Perspectives, 110 (suppl. 4), 613-618.

Levallois, P., Neutra, R., Lee. G., \& Hristova, L. (2002). Study of self-reported hypersensitivity to electromagnetic fields in California. Environmental Health Perspectives, 110 (suppl. 4), 619-623.

Lipkova, J., \& Cechak, J. (2005). Human electromagnetic emission in the ELF band. Measurement Science Review, 5(Section 2), 29-32.

McCarty, D. E., Carrubba, S., Chesson, A. L., Frilot, C., Gonzalez-Toledo, E., \& Marino, A. A. (2011). Electromagnetic hypersensitivity: Evidence for a novel neurological syndrome. International Journal of Neuroscience, 121, 670-676.

Nouri, F. M., \& Holden, J. M. (2008). Electromagnetic aftereffects of near-death experiences. Journal of Near-Death Studies, 27, 83-110.

Noyes, R., Fenwick, P., Holden, J. M., \& Christian, S. R. (2009). Aftereffects of pleasurable Western adult near-death experiences. In J. M. Holden, B. Greyson, \& D. James (Eds.), The handbook of near-death experiences: Thirty years of investigation (pp. 41-62). Santa Barbara, CA: Praeger/ABC-CLIO.

Rea, W. J., Pan, Y., Yenyves, E. J., Sujisawa, I., Suyama, H., Samadi, N., \& Ross, G. H. (1991). Electromagnetic field sensitivity. Journal of Bioelectricity, 10, 241-256.

Ring, K. (1992). The Omega Project: Near-death experiences, UFO encounters, and mind at large. New York, NY: William Morrow.

Rubin, G. J., Nieto-Hernandez, R., \& Wessely, S. (2010). Idiopathic environmental intolerance attributed to electromagnetic fields (formerly 'electromagnetic hypersensitivity: An updated systematic review of provocation studies. Bioelectromagnetics, 31, 1-11.

Schröttner, J., Leitgeb, N., \& Hillert, L. (2007). Investigation of electric current perception thresholds of different EHS groups. Bioelectromagnetics, 28, 208-213.

Smith, S. G., \& Cook, S. L. (2004). Are reports of posttraumatic growth positively biased? Journal of Traumatic Stress, 17, 353-358.

Tennen, H., \& Affleck, G. (2009). Assessing positive life change: In search of meticulous methods. In C. L. Park, S. C. Lechner, M. H. Antoni, \& A. L. Stanton (Eds.), Medical illness and positive life change: Can crisis lead to personal transformation? (pp. 31-49). Washington, DC: American Psychological Association.

Wright, B. D., \& Masters, G. N. (1982). Rating scale analysis: Rasch measurement. Chicago, IL: MESA Press.

Zingrone, N. L., \& Alvarado, C. S. (2009). Pleasurable Western adult near-death experience: Features, circumstances, and incidence. In J. M. Holden, B. Greyson, \& D. James (Eds.), The handbook of near-death experiences: Thirty years of investigation (pp. 17-40). Santa Barbara, CA: Praeger/ABC-CLIO. 